\title{
Narendra Modi's Citizen-Centered Yoga for Diabetes Management Program: Will the Indian State Install Integrative Medicine in Premier Institutes?
}

\author{
Akshay Anand \\ Department of Neurology, Postgraduate Institute of Medical Education and Research, \\ Chandigarh, India
}

More than 30,000 people were taught to perform Yoga by the Indian Prime Minister (PM) Narendra Modi at Capitol Complex in Chandigarh last summer on the occasion of the "International Yoga Day" (IYD) 2016. They not only watched their PM perform Yoga but also took his call to translate the Yoga for Diabetes management in letter and spirit. On June 21, 2016, the TV channels remained glued to Capitol Complex as Narendra Modi descended from the high-security stage, entering a special T-shirt-camouflaged, Indo-Tibetan Border Policeguarded enclosure and began following the Yoga protocol. The public suddenly got up to create space for him, duly aided by the local Deputy Commissioner. I personally experienced the aura of the PM in the same enclosure where I performed Yoga with that charismatic person. Narendra Modi's announcement of a new focus of Yoga on the IYD 2016 soon shaped the Ministry of AYUSH (Ayurveda, Yoga and Naturopathy, Unani, Siddha, and Homoeopathy)sponsored, Yoga-based Niyantrit Madhumeh Bharat (NMB) campaign ("controlled diabetic India"), which has become India's largest politico-scientific enterprise beyond Chandigarh. This discourse was quickly nationalized by none other than the PM's Yoga advisor, Dr. H.R. Nagendra, the Chancellor of SVYASA (Swami Vivekananda Yoga Anusandhan Samsthana), and the Vice-President of the Indian Yoga Association. Since then, more than 2,000 volunteers, academicians, administrators, and Yoga enthusiasts have joined the 240,000-subject study spread across the country.

The project aims at investigating the effectiveness of a diabetic protocol of Yoga in halting the transition of prediabetes into diabetes as well as the management of diabetes in general. Scientists and research scholars are upbeat about undertaking the world's biggest study, which is statistically powered to explore the horizons of Yogic lifestyle through 3 months of 
Yoga intervention in a randomly selected prediabetic and diabetic population of urban and rural India. The Yoga protocol is devised from ancient knowledge of Indian Yogic tradition. Many senior Gurus have established, Yoga-based, popular, spiritual schools, which are run all over the country. In a rare brainstorming session in Haridwar, Swami Ramdev has recently been elected as the President of the Indian Yoga Association - in a rare display of cooperation between the Guru of Art of Living, Sri Sri Ravishankar, Dr. H.R. Nagendra, Dr. Joshi, Portugal's Yoga Guru Suryananda Maharaj, and directors and representatives of the Ministry of AYUSH. This was artfully facilitated by the Quality Council of India entrusted with the job.

The focus of Yoga for Diabetes has being widely argued as part of the larger goal to install Integrative Medicine in post-modern India, where healthcare costs are escalating. A special commission, called the National Medical Commission, has been appointed by the Government of India and is all set to replace the archaic Medical Council of India. This interdisciplinary commission is likely to consider the agenda of Integrative Medicine, once the bill is passed by the Indian Parliament. The Government's goal is to provide quality healthcare at reduced costs by checking out-of-pocket expenses of patients and by promoting innovation. For diseases that are not curable or non-communicable, Yoga is re-emerging as the new health mantra, and scientists have suddenly become over-busy analyzing the new scientific evidence emerging from increased funding in Yoga research.

The NMB program has engaged about 1,200 Yoga volunteers for diabetes management (YVDM) from 380 locations in India, spanning about 62 districts from various states of India. The principal investigator, Dr. R. Nagarathna, director of Arogyadham and SVYASA, is coordinating the Yoga for Diabetics intervention program, sponsored by the Ministry of AYUSH, in the country. A special task force consisting of international experts, scientists, and Yoga masters has drawn up the grand plan. They are currently managing the resources and implementing the project in all major locations in the country. An urban and a rural location in each district have been identified before launching the door-to-door visits by trained YVDMs. The target of screening 1,000 prediabetics and diabetics and of screening follow-up confirmatory blood tests from each of the 62 districts has already been completed. The Yoga protocol is being administered to prediabetics and diabetics by new doctors (read YVDMs) who are scouting daily attendance diaries for documenting compliance. Everybody is expected to attend the early morning health mantra for 3 months.

The corresponding distant locations in the same district compile a Yoga waitlist of prediabetics/diabetics who might be initiated into Yoga at the time of IYD 2017. Almost 25-40\% of all prediabetics/diabetics in India have been reportedly discovered so far in the study. This is based on a single-page questionnaire adapted to the Indian Diabetes Risk Score (IDRS). In an unprecedented, citizen-driven Yoga-science project, the volunteers were seen carrying measuring tapes, weighing scales, and paper copies of questionnaire, frantically knocking on the doors of 240,000 households in India, visiting homes with enthusiasm, filling out forms, and collecting the participants' informed consents. Whatsapp groups became an instant hit among YVDMs, and they seamlessly communicated with other members across the country, making science fun and evidence-based in rural India. They shared challenges, experiences, resources, and pictures of blood camps that went viral on social media. The group leaders are under pressure trying to accomplish the time-bound project, regardless of their individual assignments and exams because the 3-month Yoga intervention will end on June 7, 2017. This will ensure that the reports from all locations of the country are compiled in time and analyzed before being presented to the PM just before the IYD on June 21, 2017.

Individuals participating in the project are not only excited about the free blood tests but also about becoming a subject that will generate data based on their regular Yoga practice 3 months later. Many see this as early signs of a conflict between modern and traditional medicine. A large number of the population has, however, been identified as carrying the risks 
Anand et al.: Narendra Modi's Citizen-Centered Yoga for Diabetes Management Program: Will the Indian State Install Integrative Medicine in Premier Institutes?

for diabetes. One village has been reported to be completely diabetic, while some villagers have downright rejected this project as an unimportant scheme since "they had lived their lives and would rather die than worry about impending diabetes". Detailed research papers and case reports with exciting details are likely to flood the respective journals after June 2017.

Each day, the prediabetic and diabetic individuals wait to be contacted by phone call, text message, and Whatsapp, so that they can assemble at the venue of Yoga sessions, admiring the tireless efforts of the YVDMs. They appear to be impressed by the sheer scale and social impact of the project. Each morning, from 5.30 to 6.30 a.m., people assemble at defined venues, in both rural and urban locations of India, to learn and practice the Yoga for Diabetes protocol for 1 hour. The average attendance has hovered around 80\%. The YVDMs remain busy travelling and organizing daily morning camps at multiple locations, making notes, carrying attendance-related paraphernalia from one venue to the other, so that quality data can be collected, coded, and analyzed before the IYD 2017. 